\title{
End-tidal Carbon Monoxide Levels in Prematurely Born Infants Developing Bronchopulmonary Dysplasia
}

\author{
CAROLINE MAY, SABINA PATEL, JANET PEACOCK, ANTHONY MILNER, GERRARD F. RAFFERTY, \\ AND ANNE GREENOUGH
}

\begin{abstract}
Division of Asthma, Allergy, and Lung Biology [C.M., S.P., A.M., G.F.R., A.G.], King's College London School of Medicine at Guy's, King's College and St Thomas' Hospitals, London, SE5 9RS, United Kingdom; School of Health Sciences and Social Care [J.P.], Brunel University, West London, Middlesex UB8 3PH, United Kingdom
\end{abstract}

\begin{abstract}
Bronchopulmonary dysplasia (BPD) is associated with an early inflammatory response that persists after the first week of life. Inflammatory mediators can induce hemoxygenase-1 with a consequent increase in carbon monoxide $(\mathrm{CO})$ production. End-tidal CO (ETCO) levels would be elevated in infants developing BPD. Serial measurements of ETCO levels were attempted on d 3, 5, 7, 14, 21 , and 28 in 50 prematurely born infants (median gestational age 29 wk). Fourteen infants developed BPD [oxygen dependent beyond 36 wk post-menstrual age (PMA)] and had higher ETCO levels compared with the rest of the cohort on $\mathrm{d} 7,14,21$, and 28. On d 14, the mean (SD) ETCO levels of the BPD group were 3.19 (1.11) ppm and $1.43(0.61) \mathrm{ppm}$ in the non-BPD group $(p<0.001)$. An ETCO level on $\mathrm{d} 14>2.15 \mathrm{ppm}$ had a sensitivity of $80 \%$ and specificity of $92 \%$ in predicting oxygen dependency at 36 wk PMA. Measurement of ETCO levels in prematurely born infants may be useful in the prediction of BPD. (Pediatr Res 61: 474-478, 2007)
\end{abstract}

$\mathrm{B}^{\mathrm{P}}$ PD is a common outcome of very prematurely born infants $(1,2)$. Unfortunately, it is associated with chronic respiratory morbidity including prolonged supplemental oxygen use at home (3), frequent hospital readmission (3), and troublesome respiratory symptoms (4). In prematurely born infants with respiratory distress, bronchoalveolar lavage studies have demonstrated an excess of neutrophils and alveolar macrophages in the lung effluence (5). This inflammatory reaction is compounded by such factors as oxygen toxicity and volutrauma and persists in infants who develop BPD.

Inflammatory mediators can induce an isoform of hemoxygenase (HO), an enzyme that catalyses the rate-limited oxidative cleavage of hemoglobin (6). $\mathrm{CO}$ is produced endogenously in lung tissue as a by-product of this reaction (6). Not surprisingly, then, exhaled CO levels have been shown to be increased in a number of inflammatory lung conditions including asthma, chronic obstructive pulmonary disease (COPD), and cystic fibrosis (CF) (7). Oxidative stress also significantly increases the HO-1 expression in several pulmonary cell types including macrophages, epithelial, endothelial, and fibroblast cells (8). Infants who are developing BPD are exposed to

\footnotetext{
Received June 6, 2006; accepted November 15, 2006.

Correspondence: Anne Greenough, M.D., Children Nationwide Regional Neonatal Intensive Care Centre, 4th Floor Golden Jubilee Wing, King's College Hospital, Denmark Hill London, SE5 9RS, UK; e-mail: anne.greenough@kcl.ac.uk

C.M. is supported by the Wolfson Foundation.

DOI: $10.1203 /$ pdr.0b013e3180332bfe
}

oxidative stress $(9,10)$. We, therefore, hypothesized that exhaled CO levels would be increased in prematurely born infants developing BPD. Exhaled $\mathrm{CO}$ can be assessed at the bedside by measurement of ETCO. There have been a number of studies investigating exhaled $\mathrm{CO}$ levels in neonates. These have demonstrated that exhaled $\mathrm{CO}$ levels reflect hemolysis (11) and that the ETCO level correlates closely with the corrected reticulocyte count in Coomb's test-positive term neonates (12). We can, however, find reference in a review (13) to only one study in which ETCO levels were examined in infants developing BPD. The study apparently demonstrated increased ETCO levels in infants developing BPD, but the reference is a presentation at a conference (Fanaroff A, Carbon monoxide predicts BPD, May 2003, South Eastern Association of Neonatologists Meeting, Marco Island) and no detailed information is given. The aims of this study, therefore, were to undertake sequential ETCO measurements in a population of prematurely born infants to determine whether the results were higher in infants who developed BPD and, if the levels did differ, to evaluate the predictive ability of an increased ETCO level for BPD development.

\section{METHODS}

Patients. Infants born before $32 \mathrm{wk}$ of gestation and cared for in the neonatal intensive care unit (NICU) at King's College Hospital between August 2004 and December 2005 were eligible for entry into this study if they had no congenital abnormalities and their parent(s) gave informed written consent. The study was approved by the Research Ethics Committee at King's College Hospital.

Equipment. Measurement of ETCO was performed noninvasively using the CO-STAT device (Natus Medical Inc., San Carlos, CA). The device comprised two analyzers, an electrochemical sensor to measure $\mathrm{CO}$ and hydrogen and an infrared analyzer to measure carbon dioxide $\left(\mathrm{CO}_{2}\right)$. The $\mathrm{CO}$ sensor had a time constant of $18 \mathrm{~s}$ and a time delay of $6 \mathrm{~s}$. It sampled at $1 \mathrm{~Hz}$. The $\mathrm{CO}_{2}$ sensor had a time constant of $0.3 \mathrm{~s}$ and sampled at $30 \mathrm{~Hz}$. Gas was sampled continuously during inspiration and expiration using a fine bore sampling catheter. The sampling rate was $60 \pm 6 \mathrm{~mL} / \mathrm{min}$. The signal generated from the $\mathrm{CO}_{2}$ analyzer was used for breath detection and the timing of the ETCO measurement. The software algorithm, together with a checking and averaging filter, identified the end-tidal peak in $\mathrm{CO}_{2}$. A software algorithm within the device aligned the signals from the two sensors in time to correct for the phase shift between the analyzers and identified the ETCO level. In operation, the device took $\mathrm{CO}$ and $\mathrm{CO}_{2}$ measurements after $70 \mathrm{~s}$, the period of time necessary for the $\mathrm{CO}$ analyzer to stabilize. During the subse-

Abbreviations: BPD, bronchopulmonary dysplasia; CO, carbon monoxide; ETCO, end-tidal carbon monoxide; ETT, endotracheal tube; ROC, receiver operating characteristics 
quent measurement period, the device calculated the $\mathrm{ETCO}_{2}$ level for each breath. Whenever an $\mathrm{ETCO}_{2}$ peak was located, the corresponding timecorrected CO signal was sampled. A minimum of 12 ETCO values were collected over a minimum of $20 \mathrm{~s}$ of $\mathrm{CO}_{2}$ sampling. The overall ETCO level was calculated by taking the mean of these ETCO levels. The ETCO level was then corrected for background $\mathrm{CO}$ levels through a period of ambient sampling (U.S. Patent no. 6,544,190 BI April 8th, Smits et al.). The ambient CO levels were between 0.1 and $0.6 \mathrm{ppm}$ (median $0.4 \mathrm{ppm}$ ). If no breath was detected by the $\mathrm{CO}_{2}$ analyzer and/or the hydrogen level exceeded $50 \mathrm{ppm}$, the measurement was aborted. Sampling was undertaken for $2 \mathrm{~min}$ and the results fed into an algorithm (U.S. patent no. 5,293,875) that computed the mean ETCO level. The analyzers were calibrated every $30 \mathrm{~d}$ using calibration cylinders containing $19 \mathrm{ppm} \mathrm{CO}$ and $10 \% \mathrm{CO}_{2}$.

The manufacturer states that the CO-STAT device measures $\mathrm{CO}$ over a range of $0-25 \mathrm{ppm}$ with a resolution $0.1 \mathrm{ppm}$. To determine the validity of the CO-STAT measurements, the results of the CO-STAT were compared with those of gas chromatography. Four concentrations of $\mathrm{CO}$ were assessed and three measurements were made with the CO-STAT of each concentration and the results meaned. The mean difference in the results of the CO-STAT from those of gas chromatography was $0.06 \mathrm{ppm}$ [limits of agreement 1.17-1.30, Bland and Altman analysis (14)] (Table 1).

To determine whether the sampling catheter position within the endotracheal tube (ETT) influenced ETCO results, an in vitro experiment was undertaken using a lung model. A 2.5 - $\mathrm{mm}$ Coles shouldered ETT was passed through a rubber bung inserted into the neck of a vacuum flask $(500 \mathrm{~mL})$, which acted as the lung model. Coles shouldered ETTs are used routinely on our NICU because when using such tubes, there is little or no leak around the ETT during mechanical ventilation (15). A constant flow (3 L/min) of gas [5 $\mathrm{ppm} \mathrm{CO} / 10 \% \mathrm{CO}_{2} /$ balance nitrogen (BOC special gases, Guildford, Surrey, UK)] was fed into the vacuum flask via the side arm. The ETT was connected to a ventilator circuit (SLE 5000 infant ventilator, Specialized Lab Equipment Ltd., South Croydon, UK) and positive pressure ventilation applied to the lung model using a rate of 60 breaths per minute (I:E ratio of 1:1) and peak inspiratory pressure of $20 \mathrm{~cm} \mathrm{H}_{2} \mathrm{O}$ and positive end-expiratory pressure of 4 $\mathrm{cm} \mathrm{H}_{2} \mathrm{O}$ and an inspired oxygen concentration of $30 \%$. The CO-STAT sampling catheter was passed through the suction port on the manifold of the ventilator circuit and the tip positioned at the shoulder of the ETT. The sampling catheter was then withdrawn in $1 \mathrm{~cm}$ increments across a range of $5 \mathrm{~cm}$ and sampling repeated at each position. The ETCO levels measured were between 3 and $4 \mathrm{ppm}$, reflecting that the lung model was being "ventilated" with $30 \%$ oxygen. The levels were as follows: position from shoulder within the ETT: $0 \mathrm{~cm}, 3.4 \mathrm{ppm} ; 1 \mathrm{~cm}, 3.5 \mathrm{ppm} ; 2 \mathrm{~cm}, 3.7 \mathrm{ppm} ; 3 \mathrm{~cm}$, $3.6 \mathrm{ppm} ; 4 \mathrm{~cm}, 3.1 \mathrm{ppm} ; 5 \mathrm{~cm}, 3.4 \mathrm{ppm}$. No significant differences $(p=0.07)$ were found between the $\mathrm{CO}$ levels sampled at the various sites in the ETT [analysis using one-way analysis of variance (ANOVA)]. To determine the short-term variability of ETCO measurements, repeated measurements were made on 10 babies; each baby was measured on three separate occasions over an 8 -h period. The coefficient of variability was $9.8 \%$.

Protocol. Serial measurements were attempted on d 3, 5, 7, 14, 21, and 28 after birth when the infants were stable with blood gases in a predetermined acceptable range $\left(\mathrm{Po}_{2} 7-10 \mathrm{kPa}, \mathrm{PCO}_{2} 5-7 \mathrm{kPa}, \mathrm{pH} 7.25-7.4\right)$. Measurements were not attempted in infants requiring high-frequency oscillation ventilation as the presence of the catheter within the ETT could have reduced the delivered volume by effectively reducing the internal diameter within the ETT (16). In addition, measurements were also not made in infants who were receiving nasal continuous positive airway pressure (CPAP), as end-tidal levels in $\mathrm{CO}_{2}$ could not be detected during CPAP.

In ventilated infants, the sampling catheter was passed down the ETT via the suction port on the ventilator manifold so that the tip was at the shoulder of the ETT. In nonventilated infants, the catheter tip was inserted $5 \mathrm{~mm}$ inside one nostril. Exhaled gas sampling was performed over $2 \mathrm{~min}$, after which the catheter was removed. The level of $\mathrm{CO}$ in the ambient air was then measured

Table 1. Comparison of the results of the CO-STAT to those by gas chromatography

\begin{tabular}{|c|c|c|c|c|}
\hline \multicolumn{4}{|c|}{ CO-STAT measurements } & \multirow[b]{2}{*}{ Gas chromatography } \\
\hline 1 & 2 & 3 & Mean & \\
\hline 1.9 & 1.8 & 1.8 & 1.85 & 1.82 \\
\hline 3.2 & 3.2 & 3.3 & 3.2 & 3.43 \\
\hline 15.9 & 16.1 & 16.0 & 16.0 & 16.5 \\
\hline 20.1 & 20.2 & 20.3 & 20.15 & 19.2 \\
\hline
\end{tabular}

$\mathrm{CO}$ results are in ppm. for a further 1 min and $45 \mathrm{~s}$ per the automated program, and the ETCO level was then automatically corrected for the ambient level.

Demographic data were collected from the patients' notes including information on the duration of ventilation and oxygen dependency. A number of factors potentially could influence ETCO levels including maternal smoking (17), hemolysis $(18,19)$, bilirubin levels $(20,21)$, and infection $(22,23)$. As a consequence, we recorded maternal smoking habits and on each measurement day, the serum bilirubin and hematocrit levels. In addition, it was noted whether the infants had had septic episodes. The routine policy of the NICU was to commence infants with respiratory distress at birth on antibiotics and at any time sepsis was suspected. If, however, there were no ongoing abnormal signs and blood cultures were negative, antibiotics were discontinued after $48 \mathrm{~h}$. As a consequence, infants in this study were described as having a septic episode if they had received antibiotics for at least $5 \mathrm{~d}$. Corticosteroids have been shown to reduce exhaled CO levels in asthmatic patients (24); thus, we recorded whether the infants had received corticosteroids postnatally and the number of courses per infant. The routine policy of the NICU was only to prescribe systemically administered corticosteroids to infants who remained ventilator dependent in high inspired oxygen concentrations and were at least $2 \mathrm{wk}$ of age.

Analysis. Infants were divided into two groups according to their subsequent BPD status. BPD was diagnosed if the infants remained oxygen dependent at 36 wk PMA. The data were tested to determine whether they were normally distributed using the Kolmogorov-Smirov test. The demographic data, apart from birth weight, were not normally distributed, and, therefore, differences were assessed for statistical significance using the Mann-Whitney $U$ test. The ETCO data, however, were normally distributed, and as a consequence, differences were assessed for statistical significance using one-way ANOVA and post hoc Bonferroni correction for multiple comparisons. To determine whether the ETCO level was related to the bilirubin or hematocrit level, for each measurement day Spearman's correlation coefficients were calculated. To determine whether ETCO levels were affected by septic episodes, ETCO levels of infants with and without septic episodes on each postnatal day of measurement were compared. Receiver operating characteristic (ROC) curves were constructed (25) and the areas under the curves calculated to assess whether ETCO levels and clinical variables predicted BPD development. The results on the day of measurement with the highest area under the ROC curve were further explored. Logistic regression was used to investigate the relationship between ETCO and BPD controlling for gestational age and whether the infant was ventilated at the time of the measurement. Statistical analysis was undertaken using Graph-pad PRISM 3.03 software for windows (Graph-pad Software Inc., San Diego, CA).

Sample size. The mean ETCO level in 12 babies born at term and $3 \mathrm{~d}$ old was 1.63 (SD 0.48) ppm. Recruitment of 14 babies in each group allowed us to detect a difference of $0.5 \mathrm{ppm}$ in ETCO levels between the two groups, with $80 \%$ power at the $5 \%$ level. Recruitment, therefore, continued until there were at least 14 babies who subsequently developed BPD and 14 babies who did not develop BPD entered into the study.

\section{RESULTS}

The 14 infants who developed BPD were more immature $(p<0.001)$ and weighed less at birth $(p<0.0001)$ than those who did not develop BPD; in addition, a greater proportion had received surfactant $(p=0.002)$ (Table 2). Comparison of the ETCO results of the BPD and non-BPD levels demonstrated significant differences between the groups from $\mathrm{d} 7$ onward (Table 3). There were no significant differences in ETCO levels according to maternal smoking status or any significant correlations between ETCO levels and hematocrit or bilirubin levels on any measurement day (data not shown). There were no significant differences on any postnatal age in the ETCO levels of infants with and without septic episodes at the time of measurement. On each study day, until d 28, more of the infants who developed BPD were ventilated than not, but there were no significant differences in the ETCO levels according to ventilator dependency status (data not shown). Construction of ROC curves demonstrated that ETCO results on d 14 yielded the highest area under the ROC curve (Table 4). An ETCO level on d 14 of $>2.15$ ppm had a sensitivity of $80 \%$ 
Table 2. Demographic data by BPD status

\begin{tabular}{lccc}
\hline & $\begin{array}{c}\text { BPD } \\
(n=14)\end{array}$ & $\begin{array}{c}\text { Non-BPD } \\
(n=36)\end{array}$ & $p$ \\
\hline Gestational age, wk & $26(24-29)$ & $30(24-32)$ & $<0.0001$ \\
Birth weight, g & $789(153)$ & $1330(374)$ & $<0.0001$ \\
Male, $n(\%)$ & $9(64)$ & $14(38)$ & 0.1664 \\
Antenatal steroids, $n(\%)$ & $12(86)$ & $28(78)$ & 0.533 \\
$\quad$ Doses & $1.5(0-2)$ & $1.5(0-2)$ & 0.836 \\
Surfactant, n (\%) & $13(93)$ & $16(44)$ & 0.002 \\
$\quad$ Doses & $1(0-2)$ & $0(0-2)$ & 0.013 \\
Postnatal steroids, $n(\%)$ & $2(14)$ & $1(3)$ & 0.128 \\
Septic episodes & $3(1-6)$ & $1(0-5)$ & $<0.0001$ \\
Total duration of antibiotic & $19.5(5-64)$ & $5(0-19)$ & $<0.0001$ \\
$\quad$ therapy, d & & & \\
Duration of ventilation, d & $29.5(3-101)$ & $1(0-41)$ & $<0.0001$ \\
Duration of oxygen & $92(40-289)$ & $2(0-49)$ & $<0.0001$ \\
$\quad$ dependence, d & & & \\
\hline
\end{tabular}

Data given as median (range) or number (\%).

Table 3. Serial ETCO levels according to BPD status

\begin{tabular}{crcccc}
\hline Day & $n$ & BPD & $n$ & Non-BPD & \multicolumn{1}{c}{$p$} \\
\hline 3 & 7 & $3.09(0.73)$ & 14 & $2.28(0.91)$ & $>0.05$ \\
5 & 6 & $2.75(0.42)$ & 23 & $2.24(1.51)$ & $>0.05$ \\
7 & 9 & $3.22(1.38)$ & 24 & $1.98(1.32)$ & $<0.01$ \\
14 & 10 & $3.19(1.11)$ & 25 & $1.43(0.61)$ & $<0.001$ \\
21 & 11 & $2.67(0.72)$ & 24 & $1.47(0.58)$ & $<0.01$ \\
28 & 10 & $2.53(0.81)$ & 17 & $1.31(0.50)$ & $<0.05$ \\
\hline
\end{tabular}

Data are given as mean (SD); $n$ is the number measured on a particular day. ETCO levels (in ppm).

Table 4. Areas under the ROC curves for predicting oxygen dependence at 36 wk PMA

\begin{tabular}{ll}
\hline ETCO d 3 & 0.79 \\
ETCO d 5 & 0.76 \\
ETCO d 7 & 0.81 \\
ETCO d 14 & 0.96 \\
ETCO d 21 & 0.92 \\
ETCO d 28 & 0.91 \\
Gestational age & 0.87 \\
Birth weight & 0.91 \\
Ventilated on d 14 & 0.88 \\
\hline
\end{tabular}

and specificity of $92 \%$ in predicting BPD development (Fig. 1). Logistic regression demonstrated the relationship between ETCO levels on d 14 and BPD development were not explained by gestational age or ventilation status $(p=0.05$ adjusted for gestation, ventilation not significant in the multivariate logistic model).

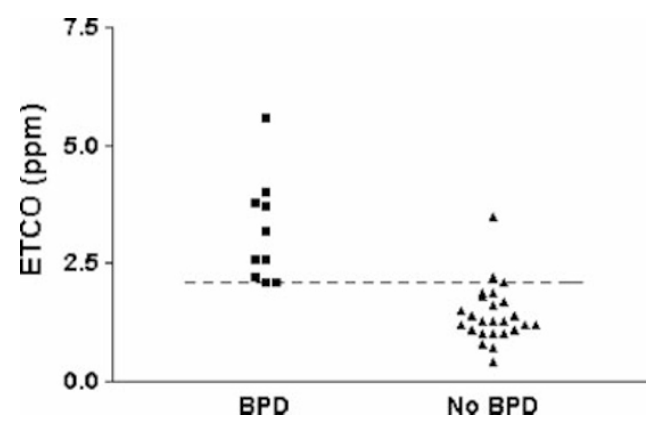

Figure 1. ETCO results on d 14 of infants who did and did not develop BPD. Individual data are demonstrated and the horizontal dashed line represents the cutoff value re sensitivity and specificity.

\section{DISCUSSION}

We have demonstrated in the first month after birth that ETCO levels from d 7 onward are significantly higher in very prematurely born infants who develop BPD compared with those who do not. In addition, we found ETCO levels on d 14 to be a sensitive and specific predictor of BPD development.

There are also a number of factors that could potentially influence ETCO results. It is possible that if the sampling rate exceeded the breathing flow, air from the surroundings could dilute the concentration in the sample chamber and result in reduced end-tidal values. The results in our in vitro study demonstrate that this was not a problem, as there was no significant change in the ETCO result as the tip of the sampling tube was moved within the ETT. If "dilution" was occurring, then the ETCO level would have become progressively lower as the tip was moved farther up the tube. It also should be noted that the measurements were made using a shouldered ETT, which we use routinely in clinical practice, and there are minimal or no leaks with such tubes (15). The infants were receiving a variety of supplementary oxygen levels. Exhaled CO levels have been shown to increase when the inspired oxygen concentration is increased in ventilated critically ill patients (26). The effect, however, was transient, and the inspired $\mathrm{CO}$ concentration was shown to be independent of the inspired oxygen fraction (26). ETCO levels are also influenced by smoking habits (27), but the infants were studied in incubators on the NICU and thus were not exposed to smoking postnatally. A number of their mothers admitted to smoking antenatally, but there were no significant differences in ETCO levels between the infants of smoking and nonsmoking mothers. ETCO levels have been shown to correlate directly with the degree of hemolysis (28). $\mathrm{CO}$ is produced in equimolar amounts with bilirubin during hemoglobin degradation, and $80 \%-90 \%$ of endogenous $\mathrm{CO}$ is a by-product of red blood cell destruction (28). Thus, it was important to determine whether increased ETCO levels in these very prematurely born infants reflected high hemolysis levels. As a consequence, on each measurement day, we measured the infants' bilirubin levels and found no statistically significant correlations of ETCO levels with bilirubin or hematocrit levels. ETCO levels can be influenced by sepsis $(22,23)$, but we saw no significant differences in ETCO levels of infants with and without septic episodes at the time of measurement. We, thus, believe that the significantly increased ETCO levels from $\mathrm{d} 7$ onward in the babies who subsequently developed BPD reflects an ongoing inflammatory reaction in the lung. ETCO levels are reduced by administration of corticosteroids (29), but no infant included in this study received corticosteroid treatment before $14 \mathrm{~d}$ of age. In addition, there were no significant differences in the numbers of infants who did and did not receive corticosteroids and between infants who did and did not develop BPD. Thus, we do not believe corticosteroid administration influenced our ETCO results in this study. There were significant differences between the infants who did and did not develop BPD, in particular, their gestational age and duration of ventilation. Regression analysis, however, demonstrated that the ETCO level was significantly 
related to BPD status independently of gestational age and ventilation.

A variety of techniques have been evaluated with regard to predicting BPD. Infants who develop BPD have abnormal lung function in the first week $(30,31)$. Comparison of the results of lung function measurements with readily available clinical data demonstrated that a low lung volume on $\mathrm{d} 2$, i.e. after surfactant therapy had been given, was the most reliable predictor of BPD development (31). Lung volume can be assessed at the bedside on the NICU using a helium gas dilution technique (31), but the equipment is complex and the measurement requires significant expertise to perform; thus, it is unlikely to be used in routine clinical practice. As a consequence, assessment of exhaled gases is an attractive option, as these measurements can be undertaken at the bedside and require only the placement of a sampling catheter either in the infant's nostril or ETT. To date, however, few data are available and studies mainly report exhaled nitric oxide (NO) results, although it has been shown possible in five ventilated preterm babies to measure exhaled breath condensate with a reduced $\mathrm{H}_{2} \mathrm{O}_{2}$ production in the first $48 \mathrm{~h}$ after birth (32). Exhaled NO levels have been shown to be increased in infants with established BPD (33) and to be reduced in such patients by administration of postnatal dexamethasone (34). Exhaled NO levels, however, were not significantly different in infants developing BPD (35). Yet we have shown CO, another inflammatory marker, was increased in such infants. Unlike NO, CO neither contains free electrons nor acts as a reactive oxygen species; the consequent greater stability of $\mathrm{CO}$ may mean that ETCO rather than exhaled NO is a more accurate measure of pulmonary inflammation, a study in which comparison of ETCO and exhaled NO results with regard to prediction of BPD is required to test that speculation. There are also practical difficulties with assessing exhaled NO levels, which are not associated with ETCO measurements. Exhaled NO levels can be contaminated with NO from the nasal and paranasal sinuses even in very prematurely born infants (36), and NO results are flow rate dependent (37). Although it was initially suggested that $\mathrm{CO}$ was released in the nose and paranasal sinus (38), in a more recent study (39), CO was not shown to be continuously released from the nasal airways (39). In addition, measurement of $\mathrm{CO}$ is flow independent (39). There are limitations to measuring ETCO with the technique we used, i.e. measurements are not possible in infants requiring high-frequency oscillatory ventilation or nasal CPAP. Nowadays, however, because of the known side effects of postnatal steroids $(40,41)$, treatment is usually only commenced after the first week after birth, as in our NICU, when few babies remain oscillator dependent (2). In addition, postnatal steroids are given to those considered at highest risk, i.e. those who remain ventilator dependent (42) Thus, in at least the majority of infants at very high risk of developing BPD and at an age when they are actively being considered for treatment with systemically administered corticosteroids, ETCO measurements can be made.

In conclusion, ETCO levels differed significantly between very prematurely born infants who do and do not develop BPD and increased ETCO levels on d 14 were a sensitive and specific predictor of BPD development. We suggest that the higher ETCO levels in the infants who developed BPD reflect an ongoing inflammatory reaction.

Acknowledgments. The authors thank Deirdre Gibbons for secretarial assistance. The CO-STAT equipment and consumables were kindly donated by Natus Medical Inc. (San Carlos CA).

\section{REFERENCES}

1. Ehrenkranz RA, Walsh MC, Vohr BR, Jobe AH, Wright LL, Fanaroff AA, Wrage LA, Poole K National Institutes of Child Health and Human Development Neonatal Research Network 2005 Validation of the National Institutes of Health consensus definition of bronchopulmonary dysplasia. Pediatrics 116:1353-1360.

2. Johnson AH, Peacock JL, Greenough A, Marlow N, Limb ES, Marston L, Calvert SA United Kingdom Oscillation Study Group 2002 High-frequency oscillatory ventilation for the prevention of chronic lung disease of prematurity. N Engl J Med 347:633-642.

3. Greenough A, Alexander J, Burgess S, Chetcuti PA, Cox S, Leeney W, Turnbull F, Shaw NJ, Woods A, Boorman J, Coles S, Turner J 2002 Home oxygen status and Rehospitalisation and primary care requirements of infants with chronic lung disease. Arch Dis Child 86:40-43

4. Thomas M, Greenough A, Johnson A, Limb E, Marlow N, Peacock JL, Calvert S 2003 Frequent wheeze at follow-up of very preterm infants: which factors are predictive? Arch Dis Child Fetal Neonatal Ed 88:F329-F332

5. Groneck P, Gotze-Speer B, Oppermann M, Eiffert H, Speer CP 1994 Association of pulmonary inflammation and increased microvascular permeability during the development of bronchopulmonary dysplasia: a sequential analysis of inflammatory mediators in respiratory fluids of high-risk preterm neonates. Pediatrics 93:712-718 6.

6. Horvath I, MacNee HW, Kelly FJ, Dekhuijzen PN, Phillips M, Doring G, Choi AM, Yamaya M, Bach FH, Willis D, Donnelly LE, Chung KF, Barnes PJ 2001 Haemoxygenase-1 induction and exhaled markers of oxidative stress in lung diseases, summary of the ERS Resesarch Seminar in Budapest, Hungary, September 1999. Eur Respir J 18:420-430

7. Kharitonov SA, Barnes PJ 2001 Exhaled markers of pulmonary disease. Am J Respir Crit Care Med 163:1693-1722

8. Camhi SL, Alam J, Otterbein L, Sylvester SL, Choi AM 1995 Induction of heme oxygenase- 1 gene expression by lipopolysaccharide is mediated by AP-1 activation. Am J Respir Cell Mol Biol 13:387-398

9. Collard KJ, Godeck S, Holley JE, Quinn MW 2004 Pulmonary antioxidant concentrations and oxidative damage in ventilated premature babies. Arch Dis Child Fetal Neonatal Ed 89:F412-F416

10. Saugstad OD 2003 Bronchopulmonary dysplasia - oxidative stress and antioxidants. Semin Neonatol 8:39-49

11. Herschel M, Karrison T, Wen M, Caldarelli L, Baron B 2002 Evaluation of the direct antiglobin (Coombs' test) for identifying newborns at risk for haemolysis as determined by end-tidal carbon monoxide concentration (ETCOc) and comparison of the Coombs test with ETCOc for detecting significant jaundice. J Perinatol 22:341-347

12. Javier MC, Krauss A, Nesin M 2003 Corrected end-tidal carbon monoxide closely correlates with the corrected reticulocyte count in Coomb's test-positive term neonates. Pediatrics 112:1333-1337

13. Harrison CM, Andersen CC 2005 Exhaled breath measure of inflammation: are they useful in neonatal chronic lung disease? Arch Dis Child Fetal Neonatal Ed 90:F6-F10

14. Bland JM, Altman DG 1986 Statistical methods for assessing agreement between two methods of clinical measurement. Lancet 1:307-310

15. Hird M, Greenough A, Gamsu HR 1990 Gas trapping during high frequency positive pressure ventilation using conventional ventilators. Early Hum Dev 22:51-56

16. Laubscher B, Greenough A, Costeloe K Performance of four neonatal high frequency oscillators Br J Intensive Care 1996;6:148-152

17. Leitch DN, Harkawat R, Askew J, Masel P, Hendrick DJ 2005 Relation of expired carbon monoxide to smoking history, lapsed time, TLCO measurement and passive smoking. Respir Med 99:32-38

18. Stevenson DK, Fanaroff AA, Maisels MJ, Young BW, Wong RJ, Vreman HJ, MacMahon JR, Yeung CY, Seidman GS, Gale R, Oh W, Bhutani VK, Johnson LH, Kaplan M, Hammerman C, Nakamura H 2001 Prediction of hyperbilirubinaemia in near term and term infants. Pediatrics 108:31-39

19. Smith DW, Hopper AO, Shahin SM, Cohen RS, Ostrander CR, Ariagno RL, Stevenson DK 1984 Neonatal bilirubin production estimated from end-tidal carbon monoxide concentration. J Pediatr Gastroenterol Nutr 3:77-80

20. Stevenson DK, Vreman HJ 1997 Carbon monoxide and bilirubin production in neonates. Pediatrics 100:252-254

21. Cohen RS, Ostrander CR, Cowan BE, Stevens GB, Hopper AO, Stevenson DK 1982 Pulmonary excermon rates of carbon monoxide using a modified technique: differences between premature and term infants. Biol Neonate 41:289-293

22. Zegdi R, Perrin D, Burdin M, Boiteau R, Tenaillon A 2002 Increased endogenous carbon monoxide production in severe sepsis. Intensive Care Med 28:793-796

23. Biernacki WA, Kharitonov SA, Barnes PJ 2001 Exhaled carbon monoxide in patients with lower respiratory tract infection. Respir Med 95:1003-1005

24. Yamara M, Sekizawa S, Monma M, Sasaki H 1999 Exhaled carbon monoxide levels during treatment of acute asthma. Eur Respir J 13:757-760 
25. Altman DG, Bland JM 1994 Diagnostic tests 3: receiver operating characteristic plots. BMJ 309:188

26. Zegdi R, Caid R, Van De Louw A, Perrin D, Burdin M, Boifeau R, Tenaillon A 2000 Exhaled carbon monoxide in mechanically ventilated critically ill patients: influence of inspired oxygen fraction. Intensive Care Med 26:1228-1231

27. Pearce MS, Hayes L 2005 Self reported smoking status and exhaled carbon monoxide results from two population based epidemiologic studies in the North of England. Chest 128:1233-1238

28. Javier MC, Krauss A, Nesin M 2003 Corrected end-tidal carbon monoxide closely correlates with the corrected reticulocyte count in coomb's test-positive term neonates. Pediatrics 112:1333-1337

29. Paredi P, Kharitonov SA, Barnes PJ 2002 Analysis of expired air for oxidation products. Am J Respir Crit Care Med 166:S31-S37

30. Greenspan JS, Abbasi S, Bhutani VK 1988 Sequential changes in pulmonary mechanics in the very low birth weight (less than or equal to 1000 grams) infant. J Pediatr 113:732-737

31. Kavvadia V, Greenough A, Dimitriou G 2000 Early prediction of chronic oxygen dependency by lung function test results. Pediatr Pulmonol 29:19-26

32. Hitka P, Cerny M, Vizek M, Wilhelm J, Zoban P 2004 Assessment of exhaled gases in ventilated preterm infants. Physiol Res 53:561-564

33. Leipala JA, Williams O, Sreekumar S, Cheeseman P, Rafferty GF, Hannam S, Milner A, Greenough A 2004 Exhaled nitric oxide levels in infants with chronic lung disease. Eur J Pediatr 163:555-558

34. Williams O, Bhat RY, Cheeseman P, Rafferty GF, Hannam S, Greenough A 2004 Exhaled nitric oxide in chronically ventilated preterm infants. Arch Dis Child Fetal Neonatal Ed 89:F88-F89
35. Williams O, Dimitriou G, Hannam S, Rafferty GF, Greenough A 2006 Lung function and exhaled nitric oxide levels in infants developing chronic lung disease. Pediat Pulmonol 42:107-113

36. Williams O, Rafferty GF, Hannam S, Milner AD, Greenough A 2003 Nasal and lower airway levels of nitric oxide in prematurely born infants. Early Hum Dev 72:67-73

37. American Thoracic Society Board of Directors 1999 Recommendations for standardized procedures for the on-line and off-line measurement of exhaled lowe respiratory nitric oxide and nasal nitric oxide in adults and children 1999 Am J Respir Crit Care Med 160:2104-2117.

38. Andersson JA, Uddman R, Cardell LO 2000 Carbon monoxide is endogenously produced in the human nose and paranasal sinuses. J Allergy Clin Immuno 105:269-273

39. Zetterquist W, Marteus H, Johannesson M, Nordval SL, Ihre E, Lundberg JO, Alving K 2002 Exhaled carbon monoxide is not elevated in patients with asthma or cystic fibrosis. Eur Respir J 20:92-99

40. Shinwell ES, Karplus M, Reich D, Weintraub Z, Blazer S, Bader D, Yurman S, Dolfin T, Kogan A, Dollberg S, Arbel E, Goldberg M, Gur I, Naor N, Sirota L, Mogilner S, Zaritsky A, Barak M, Gottfried E 2000 Early postnatal dexamethasone treatment and increased incidence of cerebral palsy. Arch Dis Child Fetal Neonatal Ed 83:F177-F181

41. Yeh TF, Lin YJ, Lin HC, Huang CC, Hsieh WS, Lin CH, Tsai CH 2004 Outcomes at school age after postnatal dexamethasone therapy for chronic lung disease of prematurity. N Engl J Med 350:1304-1313

42. Williams O, Greenough A 2003 Post-natal corticosteroid use. Eur J Pediatr 162:613 615 